\title{
Observation of Oscillatory Radial Electric Field Relaxation in a Helical Plasma
}

\author{
J. A. Alonso, ${ }^{1}$ E. Sánchez, ${ }^{1}$ I. Calvo, ${ }^{1}$ J. L. Velasco, ${ }^{1}$ K. J. McCarthy, ${ }^{1}$ A. Chmyga, ${ }^{3}$ L. G. Eliseev, ${ }^{2}$ \\ T. Estrada, ${ }^{1}$ R. Kleiber, ${ }^{4}$ L. I. Krupnik, ${ }^{3}$ A. V. Melnikov, ${ }^{2,5}$ P. Monreal, ${ }^{1}$ F. I. Parra, ${ }^{6,7}$ S. Perfilov, ${ }^{2}$ \\ A. I. Zhezhera ${ }^{3}$ and the TJ-II Team \\ ${ }^{1}$ Laboratorio Nacional de Fusión, CIEMAT, 28040 Madrid, Spain \\ ${ }^{2}$ National Research Center, Kurchatov Institute, 123182 Moscow, Russia \\ ${ }^{3}$ Institute of Plasma Physics, NSC KIPT, 310108 Kharkov, Ukraine \\ ${ }^{4}$ Max-Planck Insitut für Plasmaphysik, Greifswald 17491, Germany \\ ${ }^{5}$ National Research Nuclear University MEPhI, 115409 Moscow, Russia \\ ${ }^{6}$ Rudolf Peierls Centre for Theoretical Physics, University of Oxford, \\ Oxford OX1 3NP, United Kingdom \\ ${ }^{7}$ Culham Centre for Fusion Energy, Abingdon OX14 3DB, United Kingdom
}

(Received 13 September 2016; published 5 May 2017)

\begin{abstract}
Measurements of the relaxation of a zonal electrostatic potential perturbation in a nonaxisymmetric magnetically confined plasma are presented. A sudden perturbation of the plasma equilibrium is induced by the injection of a cryogenic hydrogen pellet in the TJ-II stellarator, which is observed to be followed by a damped oscillation in the electrostatic potential. The waveform of the relaxation is consistent with theoretical calculations of zonal potential relaxation in a nonaxisymmetric magnetic geometry. The turbulent transport properties of a magnetic confinement configuration are expected to depend on the features of the collisionless damping of zonal flows, of which the present Letter is the first direct observation.
\end{abstract}

DOI: 10.1103/PhysRevLett.118.185002

Introduction.-One of the main challenges of nuclear fusion by magnetic confinement is to confine the plasma particles and energy long enough for fusion reactions to occur in sufficient number in the central, hottest, and densest part of the confining toroidal magnetic structure. That structure consists of a strong magnetic field that is everywhere tangent to a family of nested tori, the so-called flux surfaces. The achievable central plasma pressure depends upon the characteristics of the energy transport across the flux surfaces. In axisymmetric devices like tokamaks, ion temperature gradient (ITG) driven microturbulence is a common cause of cross-field diffusion. The linear characteristics of ITG instability (namely, its large growth rates and small radial wavelengths) make it very deleterious for confinement and would pose a serious limit to the performance and economic viability of a fusion reactor. Fortunately enough, the nonlinear evolution of the instability produces zonal electrostatic perturbations (i.e., perturbations in the electrostatic potential that are constant on each flux surface) which, while not causing any

Published by the American Physical Society under the terms of the Creative Commons Attribution 4.0 International license. Further distribution of this work must maintain attribution to the author(s) and the published article's title, journal citation, and DOI. cross-field transport by themselves, reduce the radial correlation of turbulent eddies by $E \times B$ shearing [1].

The nonlinear generation of zonal flows by drift-wavetype turbulence is known to be robust [2], but the question of whether those flows were strongly damped in toroidal geometry and therefore unable to tame turbulent transport troubled the fusion community for some time [3]. In their seminal work [4], Rosenbluth and Hinton showed that long-wavelength zonal flows were not completely suppressed by collisionless Landau-type damping in axissymmetric toroidal devices like tokamaks. The initial value problem of the collisionless relaxation of a zonal electrostatic potential is known as the Rosenbluth-Hinton problem and has become a standard test for gyrokinetic codes [5].

In recent years, interest in the Rosenbluth-Hinton problem has been fostered by its extension to stellarator geometry [6-8]. Stellarators are a type of toroidal magnetic confinement device whose magnetic field is created solely by external coils. This, in turn, requires one to break the axisymmetry of the flux surfaces to achieve magnetohydrodynamic equilibrium and stability of the confined plasmas. Unlike in tokamaks, long-wavelength zonal flows are expected to be damped in stellarators even in the absence of collisions. Furthermore, their collisionless time evolution is different from that in tokamaks, in that they exhibit a low-frequency oscillation-different from the geodesic acoustic mode also observed in tokamaks-that 
accompanies the relaxation to the final residual level $[9,10]$. This oscillation constitutes a distinct feature of the collisionless electric field relaxation in threedimensional devices susceptible to experimental detection, and, at the same time, it can be more relevant for the moderation of turbulent transport than the behavior of the zonal perturbation at an infinite time.

It has been argued that the properties of the linear relaxation can influence the nonlinear saturated turbulent heat transport $[11,12]$, which would support the use of those linear properties as a computationally inexpensive figure of merit in search of turbulence-optimized magnetic configurations for future nuclear fusion reactors. In this Letter, we have taken a step further by presenting direct observations of the oscillatory relaxation of a zonal electrostatic potential perturbation in a stellarator plasma that are shown to be in qualitative agreement with gyrokinetic simulations. To our knowledge, this is the first experimental confirmation of the relevance of the linearized gyrokinetic equation to describe zonal flow relaxation in toroidal magnetized plasmas.

Theory.-The description of the collisionless relaxation of a zonal electrostatic potential perturbation makes use of gyrokinetics, the kinetic theory for strongly magnetized particles that is obtained by systematically averaging the fast quasiperiodic gyromotion of the particles around a magnetic field line to obtain a kinetic equation for the particles' gyrocenters.

In strongly magnetized fusion plasmas, the particles of any species $s$ are confined for longer than a collision time, and, consequently, their distribution function is close to a Maxwellian $f_{M s}$ with temperature $T_{s}$ and density $n_{s}$ constant on flux surfaces. In addition, the magnetization parameter $\rho_{t s} / L \ll 1$ is the basis of the scale separation assumption in the local gyrokinetic theory, based on which we can write the distribution function and the electrostatic potential in the eikonal form. Here $\rho_{t s}=v_{t s} / \omega_{s}$ is the Larmor radius of species $s$, where $v_{t s}=\sqrt{T_{s} / m_{s}}$ is the thermal speed, $m_{s}$ is the mass, $\omega_{s}=Z_{s} e B / m_{s}$ is the gyrofrequency, $e$ is the proton charge, $Z_{s} e$ is the charge of species $s$, and $B$ is the magnitude of the magnetic field $\mathbf{B}$. Finally, since the gyrokinetic equation for purely zonal perturbations is linear, we can study the evolution of a single mode. That is, using an effective radius $r(\mathbf{x})$ as the flux surface label, we can take $\varphi(\mathbf{x}, t)=\varphi_{k}(r(\mathbf{x}), t)$ $\exp \left[i k_{r} r(\mathbf{x})\right]$, where $L^{-1} \ll k_{r} \lesssim \rho_{t s}^{-1}$ and $\varphi_{k}$ varies on the scale $L$. Analogously, denoting by $F_{1 s}$ the deviation of the distribution function from $f_{M s}$, we write $F_{1 s}(\mathbf{x}, v$, $\lambda, \sigma, t)=f_{s}(r(\mathbf{x}), \theta(\mathbf{x}), \alpha(\mathbf{x}), v, \lambda, \sigma, t) \exp \left[i k_{r} r(\mathbf{x})\right]$. We are employing the magnitude of the velocity $v$, the pitch-angle coordinate $\lambda=v_{\perp}^{2} /\left(v^{2} B\right)$, and the sign of the parallel velocity $\sigma$ as coordinates in velocity space, where $v_{\perp}$ is the magnitude of the component of the velocity perpendicular to the magnetic field. Then, the gyrokinetic equation for the above kind of perturbations reads

$$
\left(\partial_{t}+v_{\|} \hat{\mathbf{b}} \cdot \nabla+i k_{r} v_{r, s}\right) h_{s}=\frac{Z_{s} e}{T_{s}} \partial_{t} \varphi_{k} J_{0 s} f_{M s},
$$

where $\quad v_{\|}=\sigma v \sqrt{1-\lambda B}, \quad \hat{\mathbf{b}}=\mathbf{B} / B, \quad$ and $\quad h_{s}:=f_{s}+$ $\left(Z_{s} e / T_{s}\right) \varphi_{k} J_{0 s} f_{M s}$. Here, $J_{0}$ is the zeroth-order Bessel function of the first kind, and $v_{r, s}=\mathbf{v}_{M s} \cdot \nabla r$, with $\mathbf{v}_{M s}$ being the magnetic drift of species $s$. We have eased the notation by writing $J_{0 s} \equiv J_{0}\left(k_{r}|\nabla r| \rho_{s}\right)$, with $\rho_{s}=v_{\perp} / \omega_{s}$. Both $f_{s}$ and $h_{s}$ vary on the scale $L$.

The solution of (1) at long times coupled to the quasineutrality equation

$$
\sum_{s} \frac{Z_{s}^{2} e}{T_{s}} n_{s} \varphi_{k}=\left\langle\sum_{s} Z_{s} \int J_{0 s} h_{s} d^{3} v\right\rangle_{r}
$$

has been derived in detail in Ref. [8]. Here, $\langle\cdot\rangle_{r}$ denotes the flux surface average. Taking the limit of small radial drift frequency in the expression for the long-time evolution of the electrostatic potential, one finds that $\varphi_{k}$ oscillates with frequency

$$
\Omega=\sqrt{\frac{A_{2}}{A_{1}+A_{0}}},
$$

where $A_{0}=\sum_{s} n_{s}\left(Z_{s}^{2} / T_{s}\right)\left\langle|\nabla r|^{2} \rho_{t s}^{2}\right\rangle_{r}, \quad A_{1}=\sum_{s}\left(Z_{s}^{2} / T_{s}\right)$ $\left\{\delta_{s}^{2}-{\overline{\delta_{s}}}^{2}\right\}_{s}$, and $A_{2}=\sum_{s}\left(Z_{s}^{2} / T_{s}\right)\left\{{\overline{v_{r, s}}}^{2}\right\}_{s}$ and where $\{Q\}_{s}:=\left\langle\int Q f_{M s} d^{3} v\right\rangle_{r}, Q$ being a function on phase space. An overline stands for the orbit average operation. We have split the radial magnetic drift into its orbit average and a component that varies along the orbit, $v_{r, s}=\overline{v_{r, s}}+v_{\|} \hat{\mathbf{b}}$. $\nabla \delta_{s}$. Observe that $\delta_{s}$ can be interpreted as the radial excursion of the particle at each point of its orbit. The oscillatory behavior of zonal flow relaxation in stellarators was originally pointed out in Ref. [10] and explained in more detail in Ref. [7]. In these references, (3) was derived, and its quantitative accuracy as a solution of (1) and (2) is proven in Ref. [13]. In Ref. [7], an approximate expression for the collisionless damping rate of the oscillations is provided as well.

From the explicit expression of the frequency (3), it is obvious why the oscillation does not exist in tokamaks, namely, because $\overline{v_{r, s}}$ vanishes identically in axisymmetric magnetic fields. Furthermore, in the derivation of this expression, one learns that the oscillation is expected to be observed if the scale of the zonal perturbation $k_{r}^{-1}$ is sufficiently large for the small radial drift frequency condition $\left|k_{r} \overline{v_{r, s}}\right| / \Omega \ll 1$ to be satisfied and sufficiently small for (1) to be applicable. It is also important to note that the oscillation with frequency (3) is a collisionless phenomenon and that introducing collisions in the system does not modify it unless the collision frequency is much larger than $\Omega$. However, the damping rate of the zonal flow is very sensitive to the collision frequency. 
Experimental setup and observations.-The hydrogen plasmas studied in this Letter were produced in the TJ-II stellarator (four periods, average field on axis $0.96 \mathrm{~T}$, edge rotational transform 1.65) and heated by $30 \mathrm{kV}$ neutral beam injection. The discharges used here have line-averaged electron densities between 1 and $2.5 \times 10^{19}$ particles $/ \mathrm{m}^{3}$ and central electron and ion temperatures of 250 and $100 \mathrm{eV}$, respectively (note that, for these temperatures, the neutral beam-produced fast ions are slowed down by Coulomb collisions with electrons). Typical profiles for these plasma conditions can be found in Refs. [14,15]. A cryogenic hydrogen pellet is injected into an otherwise effectively stationary phase of each discharge, causing a rapid change of the plasma parameters. The transient dynamics of the electric field that follows the pellet ablation is studied by means of two heavy ion beam probe (HIBP) systems $[15,16]$. A schematic view of the setup showing the relative location of the pellet injection line and the two probing systems is presented in Fig. 1. The HIBP diagnostics were set to measure plasma potential at fixed points of the plasma cross section (termed "sampled volume" in Fig. 1) with a spatial resolution of $\lesssim 1.5 \mathrm{~cm}$ and $2 \mathrm{MHz}$ sampling rate.

Figure 2 shows several diagnostic signals in a $600 \mu \mathrm{s}$ time window during a pellet injection. The ablation of the pellet, from its entry into the plasma until its complete ablation, is registered with silicon diodes that measure the line radiation (at $656.3 \mathrm{~nm}$ ) emitted from the neutral cloud that surrounds the pellet [17].

Pellets are injected with velocities of $\sim 1000 \mathrm{~m} / \mathrm{s}$ and contain between $5 \times 10^{18}$ and $10^{19}$ particles when condensed in the injector. Whereas the actual size of the pellets at first contact with the plasma edge varies, the estimated fueling efficiency is about $50 \%$. The injections cause a prompt $\sim 10 \%$ reduction of the electron and ion temperatures as the pellet travels into the central plasma and a 40\% increase of the line-averaged plasma density. The central temperatures return to their previous levels after $5 \mathrm{~ms}$, while the evolution of the line-averaged density is significantly slower (not shown in Fig. 2; see [14]). The plasma electric field undergoes a rapid evolution as observed with Doppler reflectometry in the plasma edge and the HIBP I and II in more interior positions (Fig. 2). The high time resolution and good signal-to-noise ratio of the beam probes allow us to resolve a damped oscillation in the electrostatic potential transient response, qualitatively similar to the relaxation typically observed in numerical Rosenbluth-Hinton tests in three-dimensional magnetic fields $[10,19]$.

Comparison with linear gyrokinetic simulations.-A direct comparison of the measured and simulated relaxation is shown in Fig. 3. The beginning of the simulation shows a quickly damped geodesic acoustic mode followed by the lower-frequency damped oscillation as first predicted in Ref. [10]. The plasma potential measurement is fitted (after removing the very low frequencies, below $1 \mathrm{kHz}$ ) to a damped oscillation model $\varphi_{k}(t)=A \cos (\Omega t) \exp (-\gamma t)+$ $R_{\mathrm{ZF}}$, which is also shown in the figure to highlight the

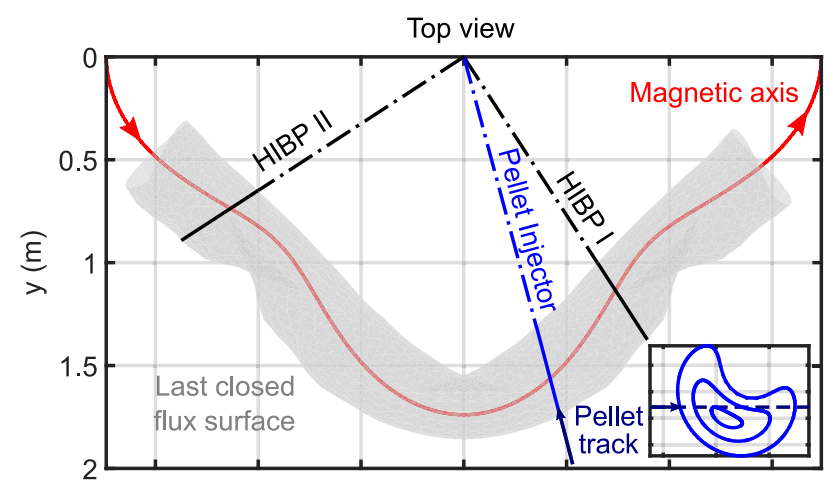

Front view

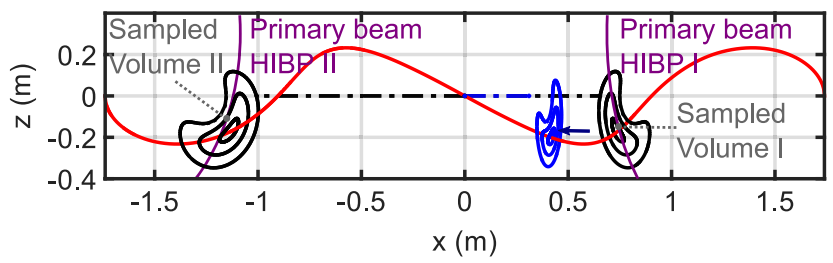

FIG. 1. Schematic view of the TJ-II magnetic geometry and experimental setup. The positions of the pellet injection line and the two HIBP systems in equal cross sections of two consecutive periods are shown. The upper plot shows a top view of one-half of the toroidal magnetic structure of the TJ-II stellarator. The magnetic axis is shown in red, and a section of the last closed flux surface is shown in gray. The inset shows the cross section of several flux surfaces at the poloidal location of the pellet injector. These and the cross sections at the location of the HIBPs are shown in a front view in the lower plot.

similarity with the result of the linear gyrokinetic simulation. The same model is used for the simulated time trace, thus obtaining pairs of experimental and simulated oscillation frequency $\Omega$ and damping rate $\gamma$.

The simulations are carried out with the gyrokinetic Monte Carlo code EUTERPE [20] in the full radial domain of the TJ-II standard magnetic configuration. The experimental density and temperature profiles together with the background radial electric field, obtained with the driftkinetic equation solver DKES [21], are used to define the equilibrium state. The run is initiated with a radially localized perturbation to the equilibrium ion probability distribution function with Maxwellian velocity dependence. The perturbed distribution function and electrostatic potential are then evolved according to the linearized gyrokinetic equations [22]. The zonal component of the electrostatic potential at the flux surface of interest is monitored, which corresponds to the simulation values displayed in Fig. 3. Both collisionless and also collisional simulations have been carried out confirming that the oscillation frequency is basically determined by collisionless processes and not much affected by collisions, while the damping rate is largely dependent on the collisionality.

To systematize and quantify the comparison shown in Fig. 3, values of the oscillation frequency and decay time are extracted from both the simulated and measured 


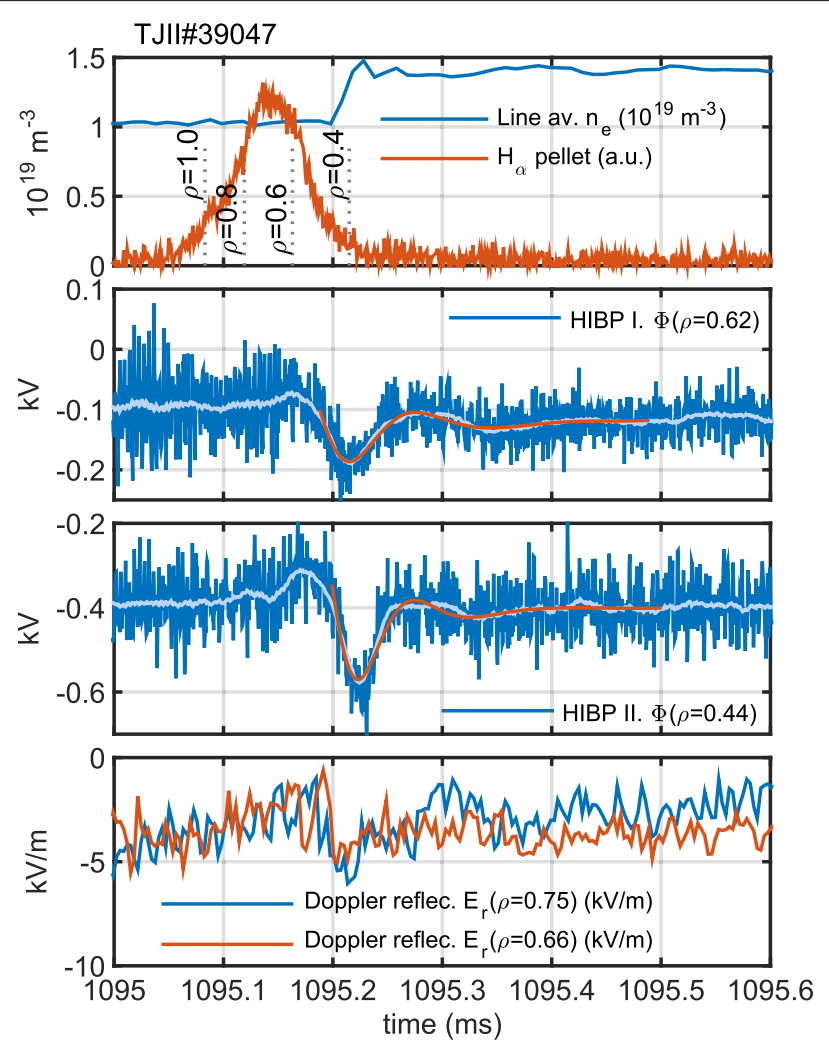

FIG. 2. Evolution of plasma parameters following a pellet injection into the TJ-II stellarator. Top: Line-averaged electron density and $H_{\alpha}$ pellet monitor. Center: Plasma electrostatic potential at two radial locations from the HIBP diagnostic. Bottom: Radial electric field in two peripheral radial locations from Doppler reflectometry [18]. In the central plots, the smoothed HIBP signals are shown in lighter blue and fits to a model damped oscillation are shown in red.

damped oscillations. This is done for the database of pellet injections in which a damped oscillation is visible. We note that not all pellet injections cause a measurable oscillation in the HIBP potentials, which is attributed to the imperfect reproducibility of the pellet and the different target plasma conditions. The results are presented in Fig. 4, with error bars corresponding to $95 \%$ confidence level in the estimation of the frequency and damping rate. The magnitude of the relative uncertainty in the fitted parameters being less than $20 \%$ was the acceptance criterion for the points in this plot. The rectangle covers the same confidence level for the parameters extracted from the decays simulated for several experimental discharges and the range of radial positions of experimental measurements. The agreement between the parameters of the simulated and measured relaxations is reasonable. In general, both the simulated damping rate and oscillation frequency show a shift towards smaller values compared to the measurement. The oscillation frequency displays a better agreement (within a factor of 2) and less dispersion than the damping rates. We note that the former, which is essentially determined by collisionless

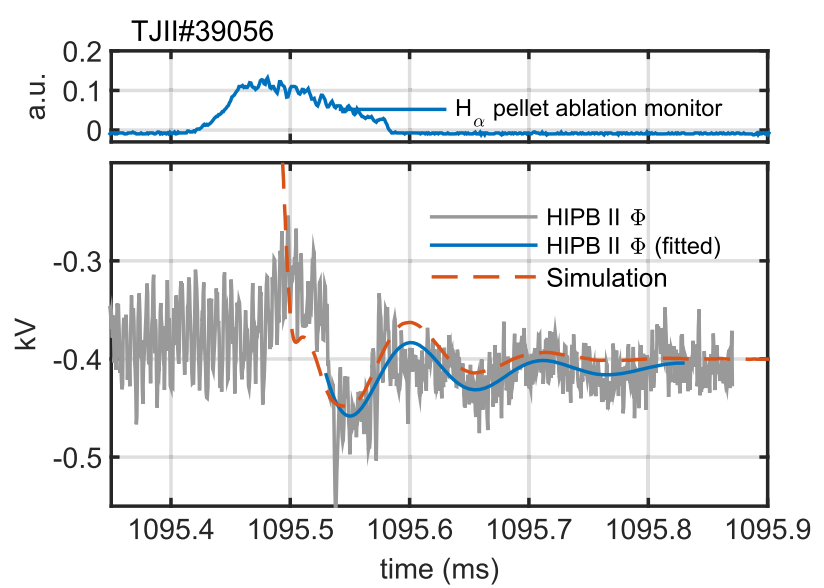

FIG. 3. Time traces of the $H_{\alpha}$ ablation monitor in a pellet injection (top) and of the electrostatic potential in a RosenbluthHinton gyrokinetic numerical simulation and in a pellet-induced transient in the TJ-II stellarator (bottom).

mechanisms, is expected to be less affected by the uncertainties in the local plasma parameters and plasma composition [23] as compared to the latter.

It might be a priori not evident why a pellet injection should trigger the characteristic short-time response of zonal flows. Both the theory and simulations show that the transient response of the electrostatic potential is a robust consequence of the system consisting of the FokkerPlanck and quasineutrality equations: One would expect to excite this oscillation rather generally, provided the particle's equilibrium distribution functions are perturbed with a sufficiently fast, global (in the flux surface) perturbation of density and/or temperature. The results here presented show that a pellet injection can reasonably provide such a fast and global perturbation, through the rapid cooldown of the electrons and the strong perturbation to the local

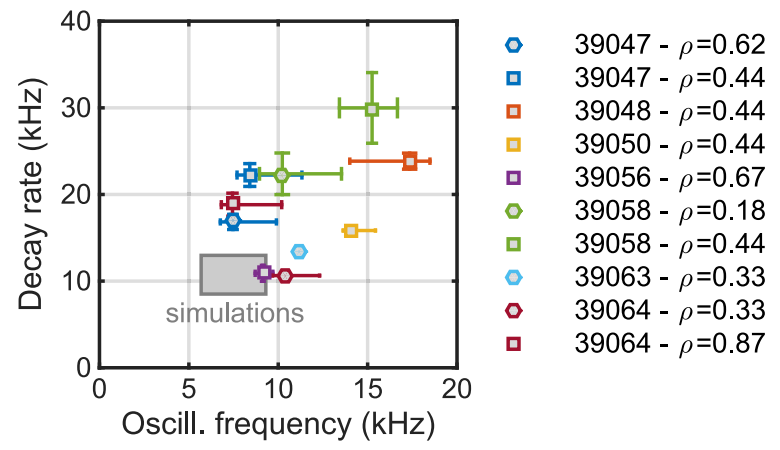

FIG. 4. Comparison of measured and simulated oscillation frequencies and damping rate for several discharges and radial positions. Measurements obtained with the HIBP I and II are labeled with circles and squares, respectively. The gray rectangle encompasses the values obtained from the simulated zonal potential relaxation for several radii in the range $0.3 \leq \rho \leq 0.9$ and five different experimental profile sets representative of the discharges. 
particle source. Its global character is supported by the fact that plasma density and temperature monitors (themselves separated in the poloidal and toroidal angle and with respect to the HIBP systems) reflect a prompt change followed by the slower, in-phase oscillation of the electrostatic potentials measured at close radial locations but two different sectors of the device (Fig. 2).

Conclusions.-In conclusion, we have presented measurements of zonal electrostatic potential relaxation in pellet-induced fast transients which are consistent with the theoretical expectations and simulations of the gyrokinetic theory. These results are important inasmuch as the nonlinear turbulence saturation level and heat transport coefficients in stellarators depend on the features of the collisionless damping of zonal electrostatic potential perturbations, of which the present Letter is the first direct observation. The methodology and observations presented here can be reproduced in existing helical devices like the recently started Wendelstein 7-X stellarator, whose magnetic configuration and low collisionality plasma conditions might allow an even clearer observation of this phenomenon.

The first author acknowledges A. Mishchenko for useful comments on the original manuscript. This work has been carried out within the framework of the EUROfusion Consortium and has received funding from the Euratom research and training program under Grant Agreement No. 633053. The views and opinions expressed herein do not necessarily reflect those of the European Commission. The work of A.V.M. was partly supported by the Competitiveness Program of National Research Nuclear University Moscow Engineering Physics Institute (NRNU MEPhI). HIBP data analysis was performed by the Kurchatov team due to the Russian Science Foundation (RSF) Project No. 14-22-00193. The activity of the Kharkov team was supported by Science and Technology Center in Ukraine (STCU) partner Project No. P507. This research was supported in part by Grants No. ENE2012-30832 and No. ENE2013-48679, Ministerio de Economía y Competitividad, Spain. The simulations were carried out within the Red Española de Supercomputación infrastructure.

[1] H. Biglari, P. H. Diamond, and P. W. Terry, Phys. Fluids B 2, 1 (1990).

[2] A. Hasegawa and M. Wakatani, Phys. Rev. Lett. 59, 1581 (1987).

[3] J. Glanz, Science 274, 1600 (1996).
[4] M. N. Rosenbluth and F. L. Hinton, Phys. Rev. Lett. 80, 724 (1998).

[5] T. Review, X. Garbet, Y. Idomura, L. Villard, and T. H. Watanabe, Nucl. Fusion 50, 043002 (2010).

[6] H. Sugama and T.-H. Watanabe, Phys. Plasmas 13, 012501 (2006).

[7] P. Helander, A. Mishchenko, R. Kleiber, and P. Xanthopoulos, Plasma Phys. Controlled Fusion 53, 054006 (2011).

[8] P. Monreal, I. Calvo, E. Sánchez, F. I. Parra, A. Bustos, A. Könies, R. Kleiber, and T. Görler, Plasma Phys. Controlled Fusion 58, 045018 (2016).

[9] The above-mentioned stronger damping of zonal flows in stellarator geometries specifically refers to the lower residual levels typically found in simulations for longwavelength perturbations.

[10] A. Mishchenko, P. Helander, and A. Könies, Phys. Plasmas 15, 072309 (2008).

[11] T.-H. Watanabe, H. Sugama, and S. Ferrando-Margalet, Phys. Rev. Lett. 100, 195002 (2008).

[12] P. Xanthopoulos, A. Mischchenko, P. Helander, H. Sugama, and T.-H. Watanabe, Phys. Rev. Lett. 107, 245002 (2011).

[13] P. Monreal, E. Sánchez, I. Calvo, A. Bustos, F. I. Parra, A. Mishchenko, A. Könies, and R. Kleiber, arXiv:1701.02722.

[14] J. L. Velasco, K. J. McCarthy, N. Panadero, S. Satake, D. López-Bruna, A. Alonso, I. Calvo, A. Dinklage, T. Estrada, J. M. Fontdecaba, J. Hernández, R. García, F. Medina, M. Ochando, I. Pastor, S. Perfilov, E. Sánchez, A. Soleto, B. P. V. Milligen, A. Zhezhera, and the TJ-II Team, Plasma Phys. Controlled Fusion 58, 084004 (2016).

[15] A. Melnikov et al., Fusion Sci. Technol. 51, 31 (2007).

[16] A. Melnikov, C. Hidalgo, L. Eliseev, E. Ascasibar, A. Chmyga, K. Dyabilin, I. Krasilnikov, V. Krupin, L. Krupnik, S. Khrebtov, A. Komarov, A. Kozachek, D. López-Bruna, S. Lysenko, V. Mavrin, J. de Pablos, I. Pastor, S. Perfilov, M. Pedrosa, R. Shurygin, V. Vershkov, and the T-10 Team, and the TJ-II Team, Nucl. Fusion 51, 083043 (2011).

[17] K. J. McCarthy et al., Proc. Sci. (2015).

[18] T. Happel, T. Estrada, E. Blanco, V. Tribaldos, A. Cappa, and A. Bustos, Rev. Sci. Instrum. 80, 073502 (2009).

[19] E. Sánchez, R. Kleiber, R. Hatzky, M. Borchardt, P. Monreal, F. Castejón, A. López-Fraguas, X. Sáez, J. L. Velasco, I. Calvo, A. Alonso, and D. López-Bruna, Plasma Phys. Controlled Fusion 55, 014015 (2013).

[20] G. Jost, T. M. Tran, W. A. Cooper, L. Villard, and K. Appert, Phys. Plasmas 8, 3321 (2001).

[21] S. Hirshman, K. Shaing, W. Van Rij, C. Beasley, Jr., and E. Crume, Jr., Phys. Fluids 29, 2951 (1986).

[22] R. Kleiber, R. Hatzky, and A. Mishchenko, Contrib. Plasma Phys. 50, 766 (2010).

[23] S. Braun, P. Helander, E. A. Belli, and J. Candy, Plasma Phys. Controlled Fusion 51, 065011 (2009). 